\title{
Differential Diagnosis of Intracranial Masses
}

\author{
James G. Smirniotopoulos and Hans Rolf Jäger
}

\section{Learning Objectives}

- Distinguish intraaxial from extraaxial and intraventricular masses

- Describe secondary effects: "volume effect" and perilesional vasogenic edema

- Distinguish metastatic disease at gray-white junction from deeper infiltrating gliomas

\subsection{Introduction}

Accurate image interpretation requires a knowledge base, understanding the patient's clinical features, and experience. Radiology is often taught as an apprenticeship of pattern recognition. However, the best interpretations use pattern analysis instead of pattern recognition: progression of clinical course (acute-rapid, subacute-smoldering, chronicprolonged); location of lesion (intra- vs. extraaxial); secondary effects (volume, edema, herniation); enhancement (solid, ring-like or leading edge, non-enhancing); blood-products; MR Spectroscopy (MRS); Diffusion-weighted Imaging (DWI and ADC values); response to treatment.

\subsubsection{Time Course of Disease}

Cerebrovascular events and traumatic lesions usually present acutely: skull impact and inertial trauma cause epidural, subdural, subarachnoid, and parenchymal hemorrhage; aneurys-

J. G. Smirniotopoulos $(\bowtie)$

Department of Radiology, Sigma Healthcare Consulting,

Silver Spring, MD, USA

\section{H. R. Jäger}

Neuroradiological Academic Unit, UCL Institute of Neurology,

London, UK

e-mail: r.jager@ucl.ac.uk mal bleeding ("thunderclap headache") into the subarachnoid space (SAH); deep hypertensive bleeds (basal ganglia and thalamus); acute neurologic deficit from ischemia-infarction (aphasia, paralysis, etc.). At the other end of the time spectrum, degenerative diseases and dementia may progress over months to years. In between, with symptom duration of weeks to months to years, are intracranial neoplasms. More rapid onset of symptoms occurs with high-grade glioma (glioblastoma) and metastatic disease.

\section{Key Point}

- Every image should be interpreted in the clinical context for each patient. When things are inconsistent, expand your differential diagnosis.

\subsection{Intraaxial Vs. Extraaxial}

Intraaxial lesions involve the parenchyma of the brain (including brainstem and cerebellum). They may follow an anatomic distribution (e.g., vascular territory for ischemia/infarction) or non-anatomic distribution (abscess). Diffuse gliomas will expand the infiltrated white-matter and tracts, causing effacement of ventricles and sulci. Extraaxial lesions will displace the brain away from the skull—often enlarging the subarachnoid cisternal spaces at the edges adjacent to the mass (Fig. 8.1).

\subsection{Pattern Analysis}

Radiology (also pathology and dermatology) are often taught using "pattern recognition." This works well once you have accumulated-through experience-a built-in library of "patterns" that you may match with an unknown case. Another approach is a break down of the imaging features, analyze them separately, and then synthesize a differential diagnosis. Patterns of contrast enhancement may suggest 

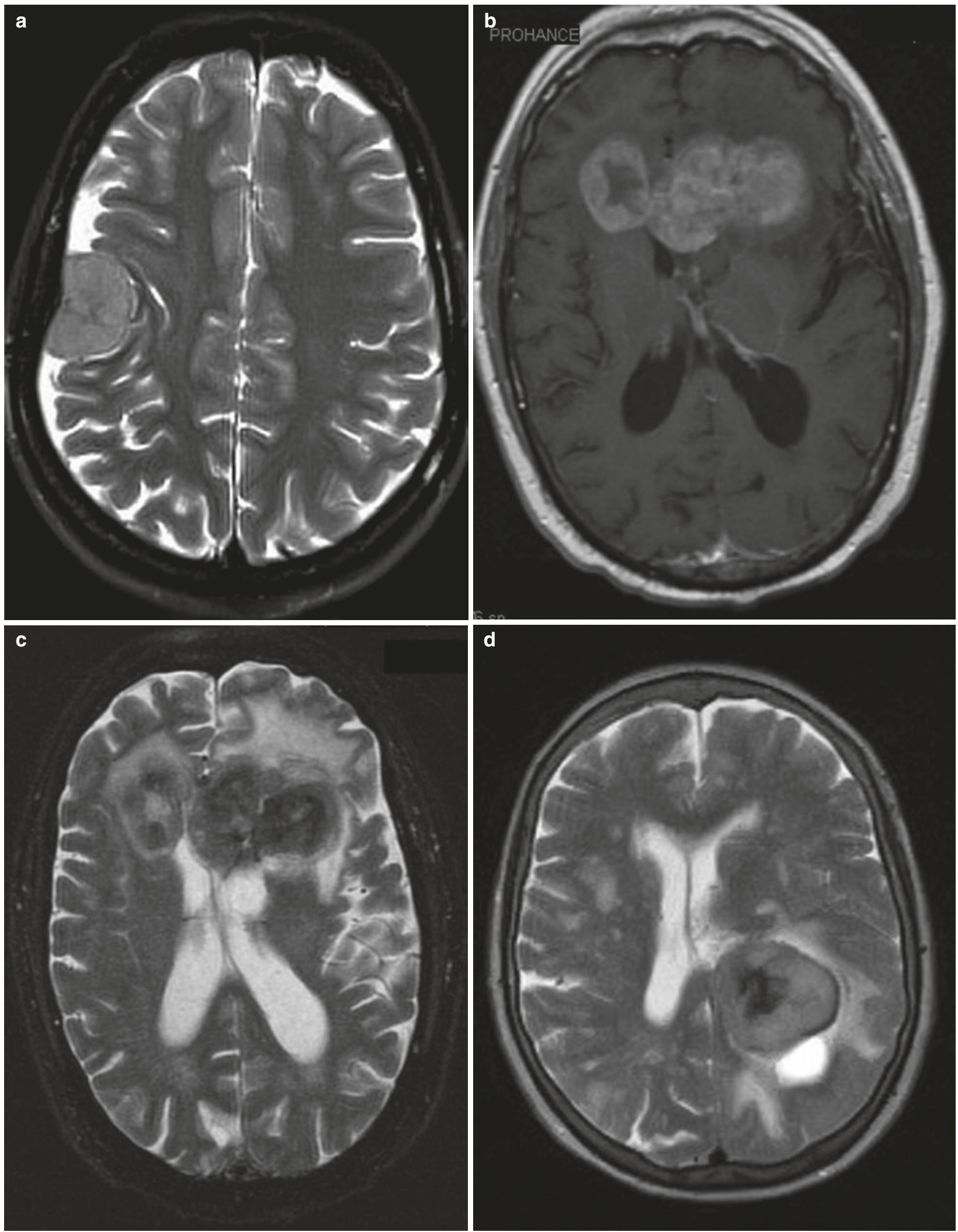

Fig. 8.1 (a) Extraaxial mass (meningioma). Axial T2-weighted MR shows a right-side gray-matter signal-intensity hemispheric-shape mass with enlargement of the SAS at its margins. There is underlying hyperostosis. (b, c) Intraaxial primary CNS lymphoma. Axial T1-weighted with gadolinium (b) shows an enhancing intraaxial mass in the genu of the corpus callosum. On the T2-weighted image (c), the lesion shows lower signal (restricted diffusion) and perilesional vasogenic edema in

the white-matter and sparing the cortical gray-matter. Infiltrating expansile lesions of the corpus callosum are usually diffuse glioma or primary CNS lymphoma. (d) Intraventricular meningioma. Axial T1-weighted image shows a rounded lesion in the trigone of the left lateral ventricle. Ventricular meningiomas arise from arachnoid cells within the choroid plexus, and the trigone is the most common location 
more specific diagnoses: gyral gray-matter enhancement occurs with reperfusion after ischemia, post-ictal or seizures, and meningoencephalitis; ring enhancement implies "central necrosis"- but may be seen with an organized abscess as well as neoplasms [1].

\subsection{Neoplastic Vs. Non-neoplastic Disease}

Neoplastic diseases are usually "tumefactive" - they take up space ("positive volume effect") and displace adjacent structures causing secondary damage and herniation. Both primary and secondary (metastatic) lesions may have added volume from accumulation of interstitial fluid-brain edema-most often due to abnormal increased permeability from breakdown of the blood-brain-barrier (BBB): vasogenic edema. In contrast, inflammatory demyelination often has minimal mass effect - it's "volume neutral". Chronic destructive processes (demyelination, healed viral infections, old infarcts) have "negative volume effect" (Fig. 8.2).

\section{Key Point}

- Chronic destructive lesions cause a loss of brain volume or "negative volume effect," with compensatory enlargement of ventricles and sulci. Adding cells (blood, pus, neoplasm) usually cause proportionate "positive volume effect" with compression of ventricles and effacement of sulci.
Tumefactive demyelinating lesions (TDL) are a frequent differential dilemma. If correctly identified, they should not be biopsied. Instead, a trial of immune-modulating therapy should be given. Features suggesting a TDL (instead of a neoplasm) include: lesion centered in white-matter (including corpus callosum); central-vein sign; "garland-like" or incomplete rim-enhancement ("open ring" or "horseshoe"); less than expected volume effect; rCBV lower than neoplasm; ADC values higher than neoplasm (GBM or lymphoma); and non-contrast CT showing low attenuation (compared to solid portions of GBM and hyper-attenuation of lymphoma [2-4] (Fig. 8.3).

\section{Key Point}

- Tumefactive demyelination may be distinguished from a neoplasm by paucity of mass-effect; mini$\mathrm{mal} / \mathrm{no}$ perilesional edema; "open ring" enhancement; increased ADC; and low perfusion values.

\subsection{Single Vs. Many Lesions}

The vast majority of intracranial lesions may present as a single/solitary mass: primary neoplasm; hematoma; abscess; infarction. Multiple lesions primarily occur as part of a systemic disease: inflammatory; toxic; metabolic; genetic; or hematogenous dissemination (Fig. 8.4). Hematogenous dissemination of infection and metastatic neoplasms can pres-
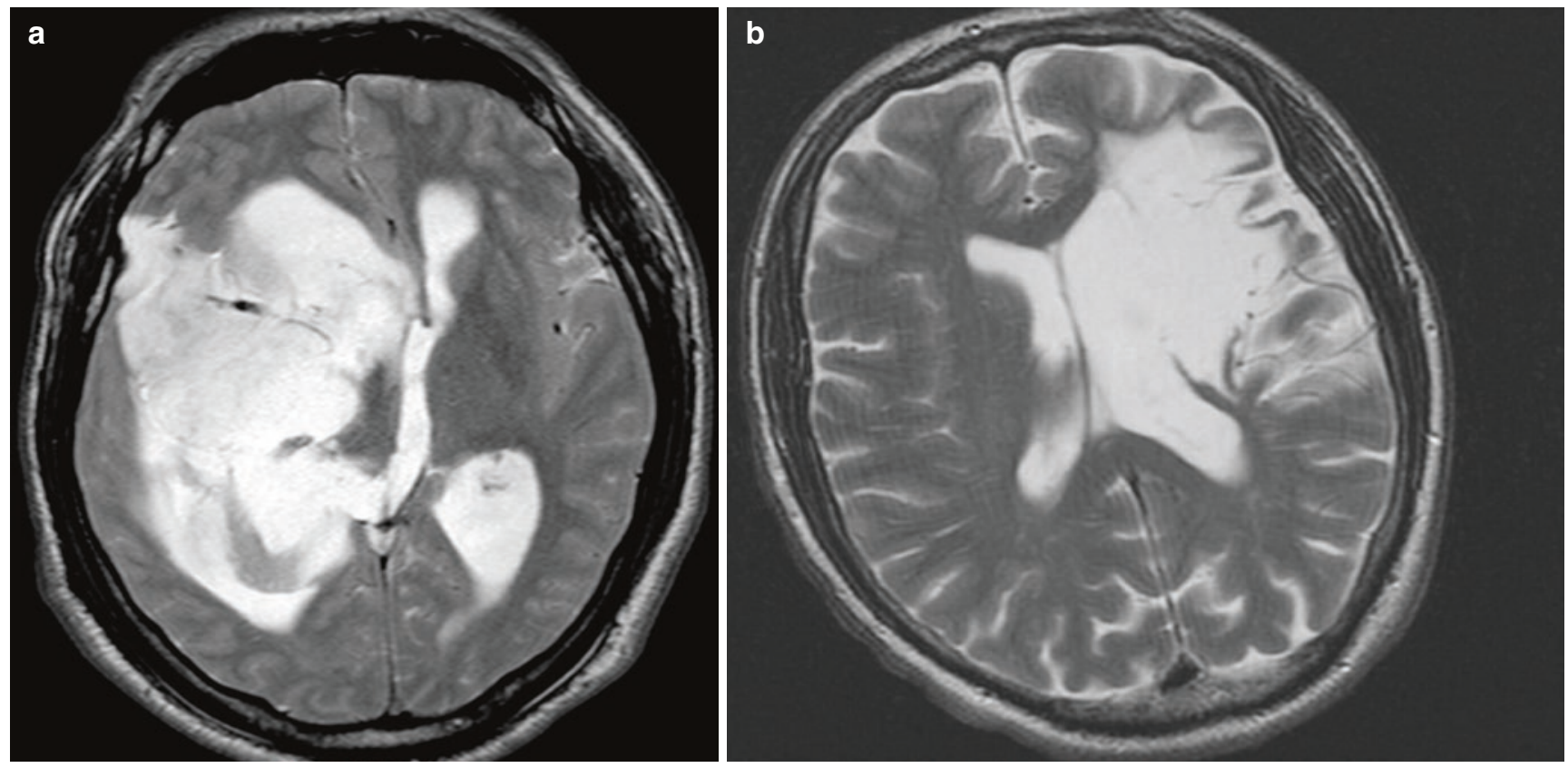

Fig. 8.2 (a) Oligodendroglioma. Axial T2-weighted image shows a single, large, deep, expansile lesion effacing both the cortical sulci and the adjacent ventricle. A lesion of this size, to present so late in its natural history, must be both slowly growing and non-destructively infiltrating. Positive "volume effect." (b) Chronic cerebral infarction. Axial T1-weighted image shows a large region of hyperintensity in the territory of the left middle cerebral artery. There is compensatory enlargement of the adjacent ventricle, overlying sulci, and subarachnoid space. Negative "volume effect" 


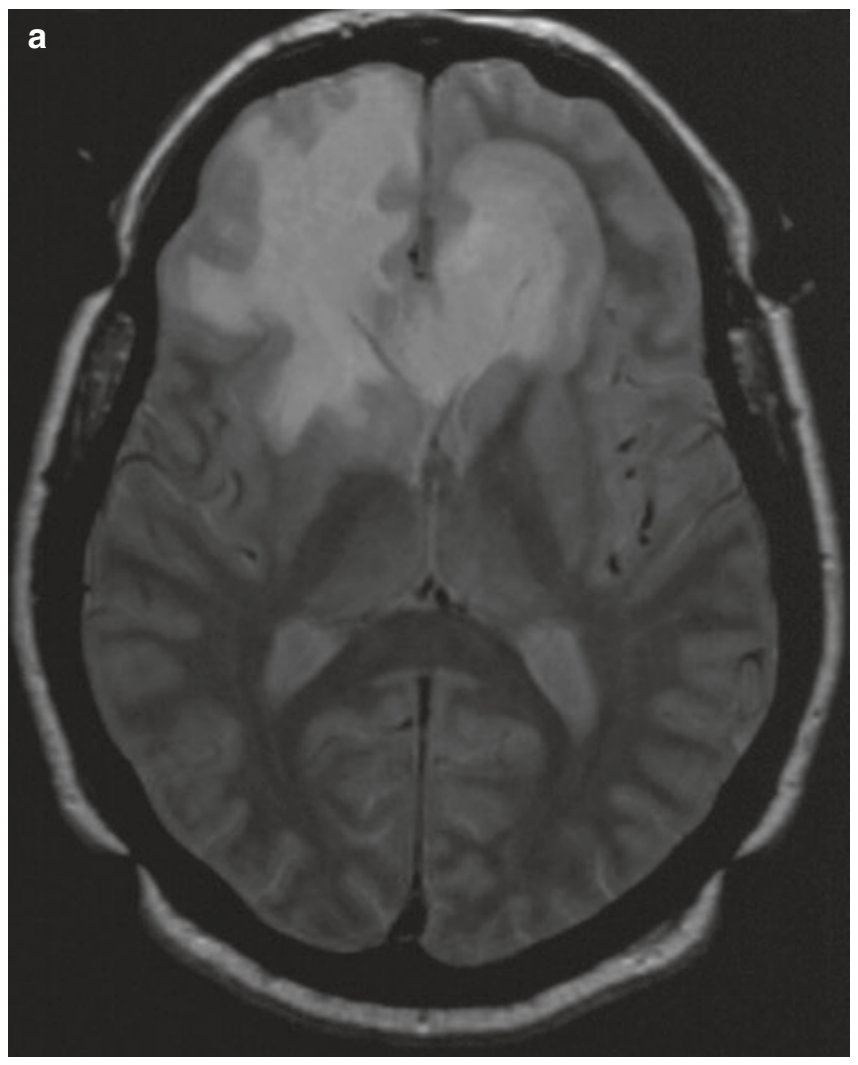

Fig. 8.3 (a, b) Tumefactive Demyelination (path-proven). Axial proton-density (a) and T1-weighted MR with gadolinium (b) show a large expansile lesion of the corpus callosum, extending into both frontal lobes. This is strongly suggestive of a diffuse high-grade astrocy-

ent with solid nodular as well as ring-enhancing lesions. Approximately $40-60 \%$ of patients with hematogenous metastasis will present initially with a single or solitary metastasis [5]. Inflammatory and auto-immune diseases (without infection) typically show multiple lesions: multiple sclerosis-MS, acute disseminated encephalomyelitisADEM (Fig. 8.4e).

\section{Key Point}

- Multiple lesions have many possible causes-some neoplastic, others toxic, metabolic, inflammatory, or genetic.

\subsection{Metastatic Disease}

Solid tumors require angiogenesis to enlarge beyond a couple of millimeters. The bio-machinery to grow new vessels can remodel existing vessels and allow embolization of tumor cells. Once in circulation, the tumor emboli are distributed by blood flow: hematogenous dissemination. The

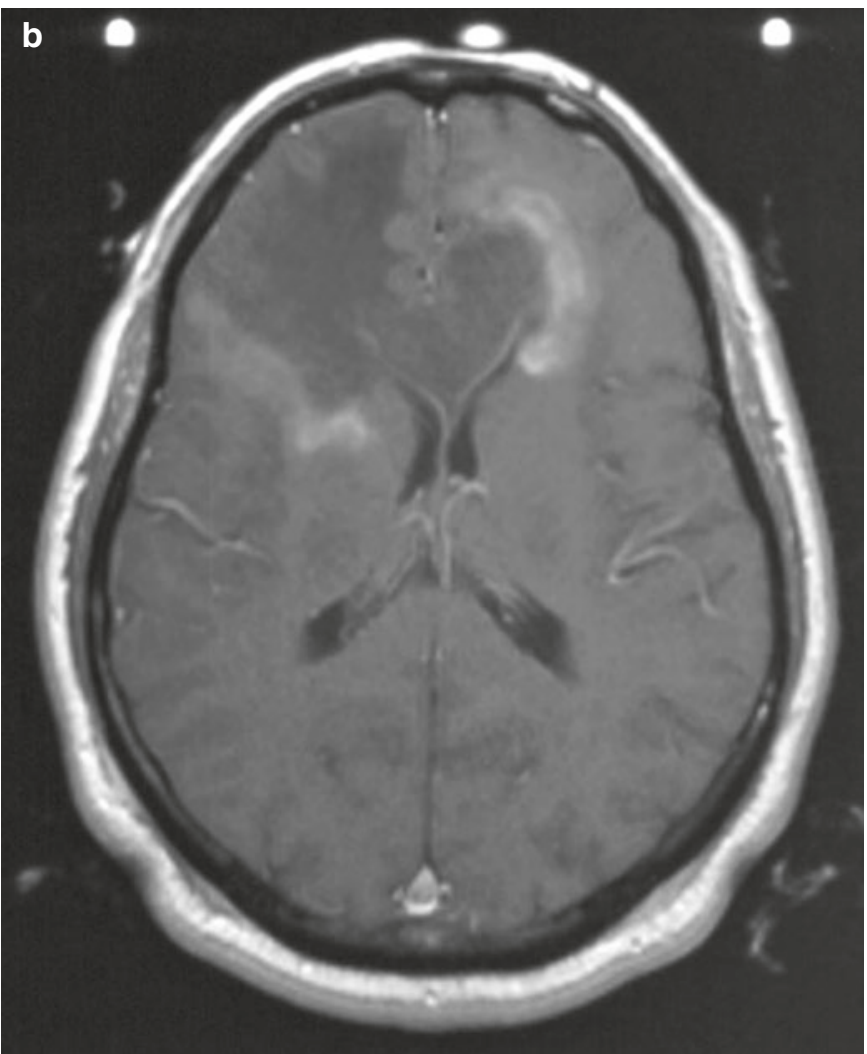

toma (glioblastoma). However, there is discontinuous peripheral "garland-like" enhancement-more characteristic of inflammatory demyelination. The appearance was so suggestive of neoplasm that a biopsy was performed

brain requires about $20 \%$ of our cardiac output. It is a vast "filtration bed" where platelet thrombi, clots, and tumor emboli may lodge. The majority of these "particulates" will lodge in two places: in the subcortical gray-white junction, and in the penetrating vessels that supply the deep graymatter (Fig. 8.4b, c) [6] Within the brain parenchyma, metastases are remarkably well localized, round solid or ring enhancing, often with a rim of reactive gliosis, and surrounded by perilesional vasogenic edema (Fig. 8.4d). Metastases from highly cellular primary tumors (such as small cell carcinoma) may show restricted diffusion. Survival after presentation with parenchymal brain metastasis may be as short as 6 months.

\section{Key Point}

- Metastatic lesions are usually peripheral cortical or subcortical, round, and well-demarcated with enhancement and perilesional vasogenic edema. At initial presentation with metastatic disease, $40-60 \%$ of patients will have a single or solitary (only brain) metastatic lesion. 

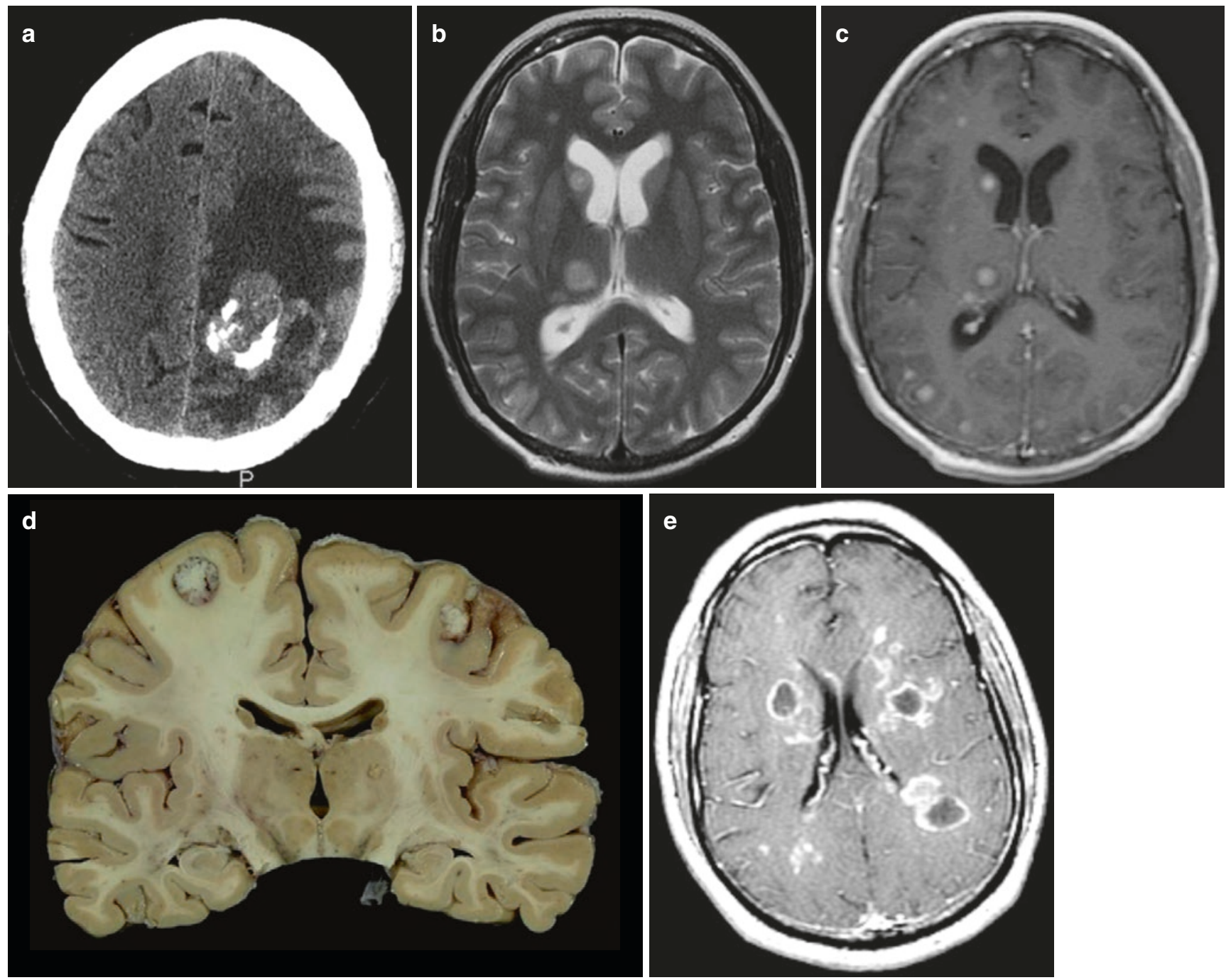

Fig. 8.4 (a) Hemorrhagic glioblastoma (WHO Gr4). Axial noncontrast CT shows a heterogenous lesion with multi-focal high attenuation (clots), surrounded by vasogenic edema in the white-matter and preservation of normal cortical gray-matter. Vasogenic edema spares the gray-matter. Single lesion. (b, c) Metastatic breast carcinoma. Axial MR (b) T2-weighted and (c) T1-weighted with gadolinium show multiple solid nodular lesions in the deep gray-matter (caudate, putamen, and thalamus) as well as multiple cortical/subcortical enhancing lesions. Multiple lesions, bilateral, but asymmetric. (d) Metastatic breast cancer (different patient). Coronal gross brain. There is a solitary, sharply demarcated and round lesion in the subcortical white-matter near the gray-white junction of the high convexity of the left hemisphere. Metastasis in the brain is usually round and focal. (e) ADEMAcute disseminated encephalomyelitis. Axial T1-weighted after gadolinium. There are multiple ring-enhancing lesions. Notice they are centered in the white-matter, and they are associated with smaller nodular enhancing lesions. ADEM is usually a monophasic process associated with an immune stimulus (infection or vaccination) primarily in children and young adults. In many cases - but not this one-we would see many "open ring" lesions. This appearance is concerning for abscesses-like toxoplasmosis. However, the patient was not immune suppressed

\subsection{Primary Neoplasms}

\subsubsection{Extraaxial Neoplasms}

Extraaxial neoplasms arise from the supporting tissues of the meninges (meningioma) and the nerves passing from central to peripheral (schwannoma). These are the two most common extraaxial and most common non-glial tumors. Meningiomas occur anywhere around the cerebral convexi- ties, along the falx and tentorium, and on the skull base. Meningiomas arise from the arachnoid and usually have a broad base of attachment to the adjacent dura — giving them a hemispheric or "globose" shape (Fig. 8.5a). A second morphology is the "en plaque" meningioma-a thickening growing along the inner table of the skull like a flat bread (pita bread) (Fig. 8.5b). Meningiomas usually maintain a homogenous CT high attenuation, and MR gray-matter-like signal-intensity, and enhancement as they grow larger (Fig. 8.5). They are commonly associated with adjacent 

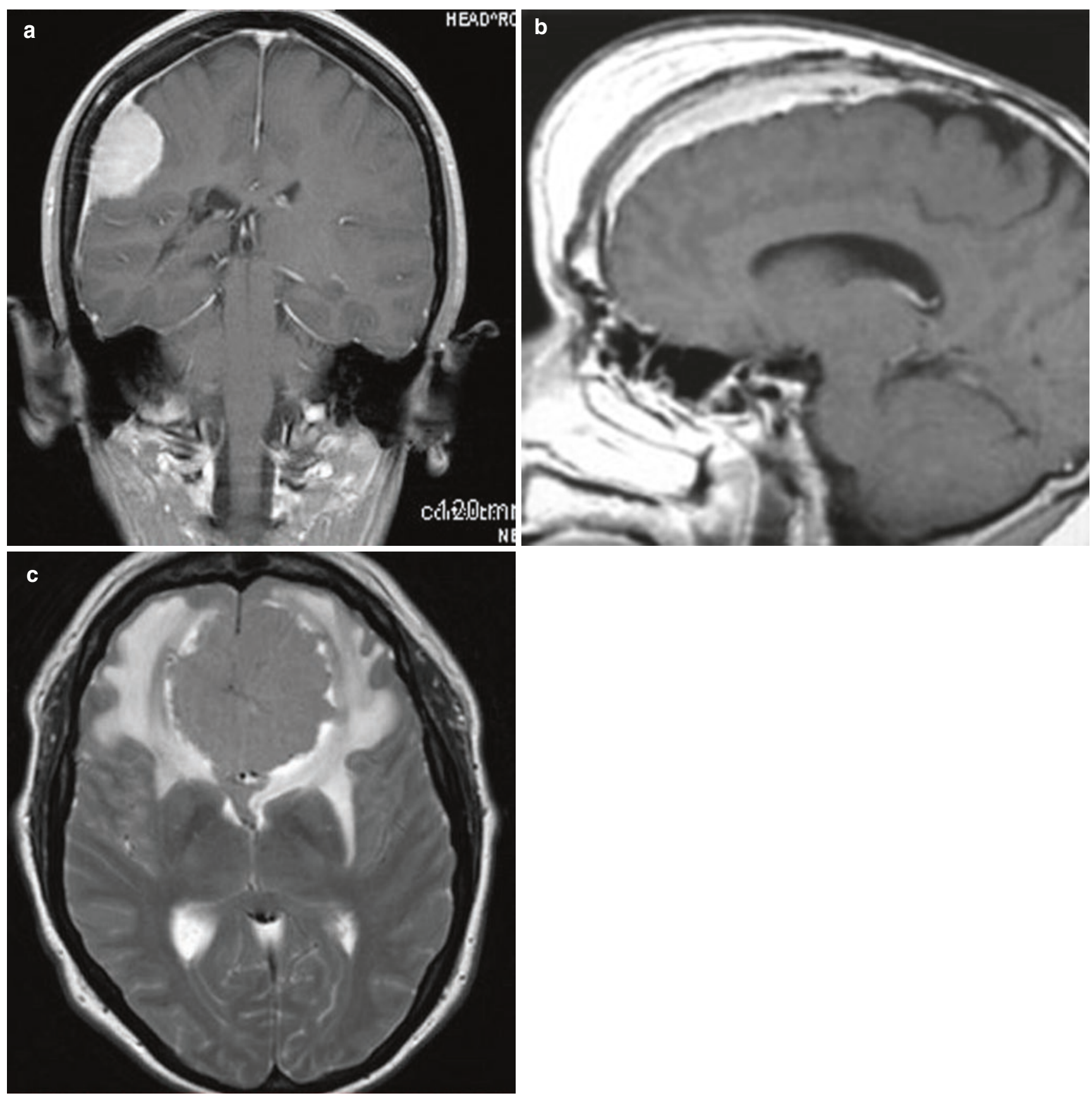

Fig. 8.5 (a, b) Meningioma. (a) Coronal T1-weighted MR after gadolinium This globose meningioma shows a sharply demarcated, hemispheric, extraaxial lesion with overlying calvarial thickening (hyperostosis). The nearby adjacent dura is slightly thickened with avid enhancement - the "dural tail." This represents a reactive process, not neoplastic infiltration. (b) Sagittal T1-weighted MR after gadolinium. This en plaque meningioma has invaded through the skull, forming a large scalp mass. These disturbing imaging features do not correlate

hyperostosis and an enhancing "dural tail" [7]. Intraaxial vasogenic edema occurs in the majority of meningiomas and is only loosely correlated with histology [8]. It is more often seen when the tumor does not have a clear visible CSF-cleft; has high signal-intensity on T2; and, a pial blood

with up-grade in histology - this was a WHO grade 1 tumor. (c) Meningioma. (c) Axial T2-weighted MR shows a bi-frontal soft-tissue mass centered on the falx, with non-specific gray-matter signalintensity. There is bi-frontal intraaxial vasogenic edema that skirts the gray-matter of the lenticular nuclei. Meningiomas easily infiltrate through the dura (not a sign of malignancy)—while other lesions (metastasis) usually do not

supply (rather than dural-only) [9]. On perfusion imaging, they usually have increased $\mathrm{rCBV}$ and $\mathrm{rCBF}$ - while showing prolonged MTT (Meant Transit Time). This is the MR equivalent of a prolonged tumor blush and delayed venous filling. On routine MRS about $1 / 2$ of well-differentiated 
meningioma will show an alanine peak (1.35-1.55 ppm), and, at 3T, there is a "characteristic" glutamine/glutamate MRS peak at $3.8 \mathrm{ppm}[10]$.

An important differential diagnosis of "en plaque" meningiomas are dural metastases in the appropriate clinical setting (e.g., in a patient with CA breast).

Schwannomas arise from the peripheral portions of the cranial nerves [3-12] - so they are ventral to the brainstem, most commonly in the posterior fossa. The most common site of origin is the inferior division of the vestibular nerve, at the apex of the internal auditory canal (IAC)_VS (vestibular Schwannoma). The tumors will compress the adjacent cochlear nerve-causing high-frequency hearing loss, but vestibular dysfunction may be retained. They grow out of the IAC into the cerebellopontine angle (CPA) cistern-where they present the bulk of the mass (Fig. 8.6). Like meningiomas VS will show avid enhancement. However, unlike meningioma, large Schwannoma become heterogenous from benign cystic degeneration. There are no imaging signs that reliably predict tumor growth, but larger tumors at presentation tend to grow faster [11].

\section{Key Point}

- Meningiomas usually remain homogeneous, even when large. Vestibular schwannomas are distinguished by location at a nerve (e.g., arising within the internal auditory canal), and they often become heterogeneous as they grow and enlarge. Almost all extraaxial and non-glial neoplasms show contrast enhancement.

\subsubsection{Intraaxial Neoplasms}

The overwhelming majority of primary intraaxial neoplasms arise from the glia—or glial cell precursors.

After glial tumors, primary CNS lymphoma (PCNSL) represents an important intrinsic neoplasm.

There are several types of glioma-usually categorized as circumscribed or diffuse (Table 8.1). The most frequent glioma in adults is, unfortunately, glioblastoma multiforme (GBM) which has the worst prognosis-median survival 12-15 months. With the exception of intraventricular neoplasms (ependymoma, choroid plexus tumors), most gliomas
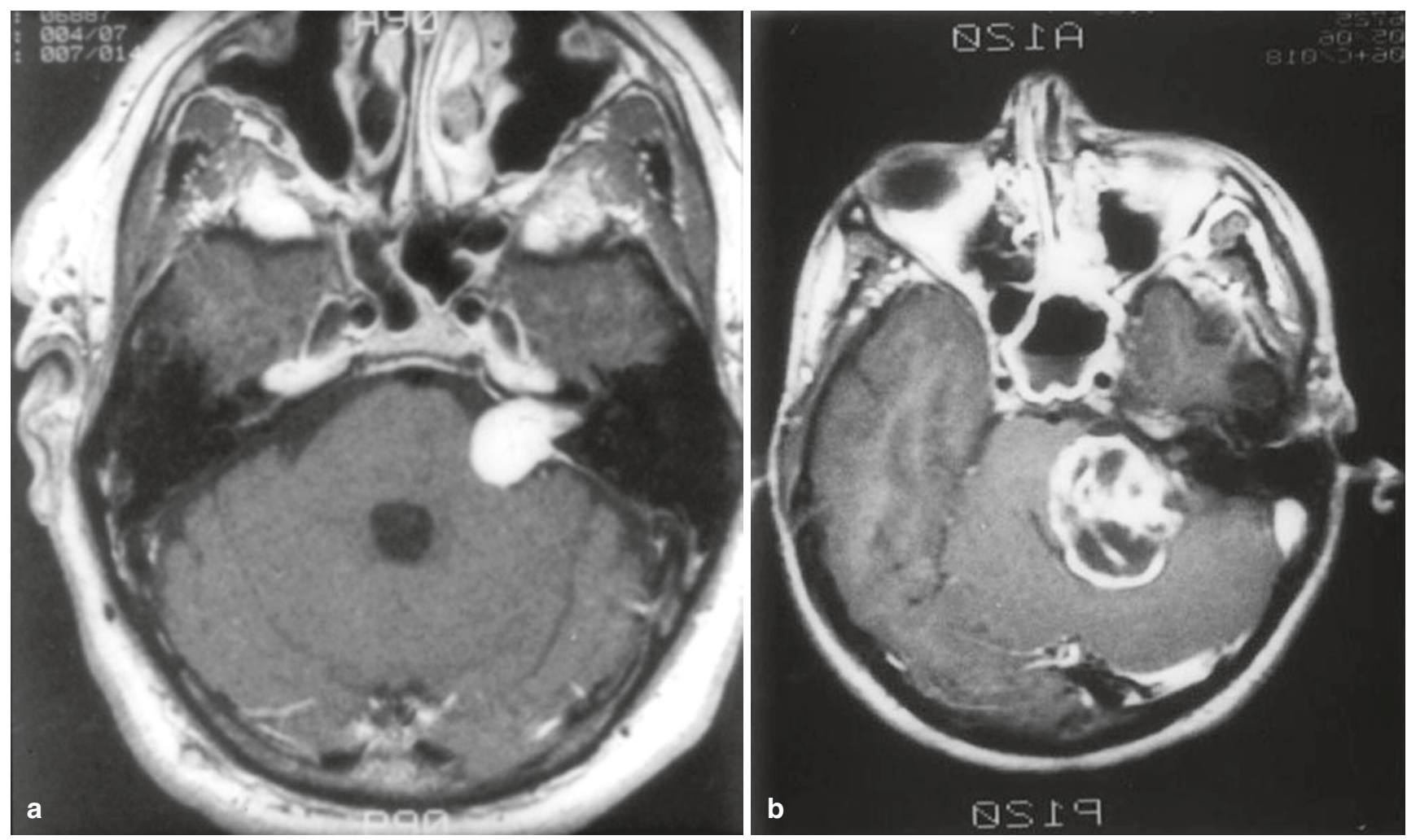

Fig. 8.6 (a) Vestibular Schwannoma. Axial T1-weighted MR after gadolinium shows a homogeneously enhancing left-sided mass in the cerebello-pontine angle cistern with a conical component inside an enlarged internal auditory canal. Note: there is a small "dural tail" along the posterior margin of the mass. The "dural tail" is a reactive process that may occur with any lesion abutting the dura. (b) Vestibular schwan- noma. Axial T1-weighted MR after gadolinium. Large extraaxial mass in the cerebello-pontine angle cistern. This is an "older" schwannoma that has become heterogeneous from benign cystic degeneration. If this lesion were incorrectly localized as intraaxial, the complex morphology would suggest a glioblastoma 
Table 8.1 Glial and mixed neoplasms

\begin{tabular}{|c|c|c|}
\hline \multirow[b]{2}{*}{ Circumscribed glioma } & \multicolumn{2}{|c|}{ Diffusely infiltrating gliomas } \\
\hline & Lower-grade diffuse & High-grade diffuse \\
\hline $\begin{array}{l}\text { Pilocytic astrocytoma } \\
\text { Gr1 }\end{array}$ & $\begin{array}{l}\text { Astrocytoma IDH-wt } \\
\text { Gr2 }\end{array}$ & $\begin{array}{l}1 \text { Glioblastoma } \\
\text { IDH-wt Gr4 }\end{array}$ \\
\hline $\begin{array}{l}\text { Subependymal giant-cell } \\
\text { astrocytoma Gr1 }\end{array}$ & $\begin{array}{l}\text { Astrocytoma } \\
\text { IDH-mut Gr2 }\end{array}$ & $\begin{array}{l}2 \text { Glioblastoma } \\
\text { IDH-mut Gr4 }\end{array}$ \\
\hline $\begin{array}{l}\text { Pleomorphic } \\
\text { xanthoastrocytoma Gr2,3 }\end{array}$ & $\begin{array}{l}\text { Anaplastic Astro } \\
\text { IDH-wt Gr3 }\end{array}$ & \\
\hline $\begin{array}{l}\text { Pilomyxoid } \\
\text { astrocytoma Gr2 }\end{array}$ & $\begin{array}{l}\text { Anaplastic Astro } \\
\text { IDH-mut Gr3 }\end{array}$ & \\
\hline Ganglioglioma Gr1 & & \\
\hline Ependymoma Gr1,2,3 & $\begin{array}{l}\text { Oligodendroglioma } \\
\text { Gr2 }\end{array}$ & \\
\hline $\begin{array}{l}\text { Choroid plexus } \\
\text { neoplasms (papilloma, } \\
\text { carcinoma, etc.) Gr1,2,3 }\end{array}$ & $\begin{array}{l}\text { Anaplastic } \\
\text { oligodendroglioma } \\
\text { Gr3 }\end{array}$ & \\
\hline
\end{tabular}

$G r$ WHO grade, mut mutant, $w t$ wild-type

Note: Oligodendrogliomas are invariably IDH-mut and 1p19q co-deleted

are large lesions that arise in the deeper periventricular white-matter-only rarely in the gray-matter (most often in the thalamus) (Fig. 8.7) Diffuse gliomas follow the whitematter tracts-using them as a scaffolding for spreadextending horizontally through the corpus callosum, vertically via cortico-spinal tracts, and through the association tracts within a single hemisphere (Fig. 8.7). Diffuse gliomas may also extend toward the cortical surface and infiltrate the gray-matter. The perilesional signal abnormality - seen best on FLAIR and T2-weighted images-is a mixture of vasogenic edema with infiltrating tumor cells. However, since vasogenic edema will not involve the cortex, cortical signal changes represent neoplastic infiltration.

\section{Key Point}

- Cortical gray-matter signal changes with a large white-matter lesion suggest neoplastic infiltration-a differential feature favoring diffuse gliomas, not seen with metastasis nor tumefactive demyelination.

Gliomagenesis for diffuse gliomas follows several different pathways. Up to $80 \%$ of lower-grade (WHO Gr 2-3) diffuse glioma have a gain-of-function "priming mutation" in IDH (isocitrate dehydrogenase-90\% IDH1 and $10 \%$ IDH2) that produces a 10,000 -fold increase in dextro-2-hydroxygutarate (D2HG) - that can be used as a tumor marker on 3T MRS [12]. Astrocytoma adds mutations in TP53 and ATRX. Oligodendrogliomas begin with the same IDH mutation, but add other mutations (TERT) and a codeletion of $1 \mathrm{p} 19 \mathrm{q}$, which is now the de facto standard marker [13]. Glioblastoma (GBM) arises from a more complex series of mutations: $90 \%$ are IDH-wild type (primary GBM, usually older patients. However, about $10 \%$ of GBM are secondary, arising from a prior lower-grade lesion that has transformed. These are usu- ally IDH-mutated and present in younger patients, often $<40$ years old.

Lower-grade diffuse gliomas (WHO Gr2,3) are deeply infiltrating masses without necrosis. They expand and nondestructively spread through the brain-often delaying the onset of a neurologic presentation. Diffuse lower-grade astrocytoma (WHO Gr 2,3) is usually homogeneous low attenuation on CT with water-like signal-intensity on MR. WHO Gr 2 astrocytomas do usually do not enhance, because they do not produce neovascularity. Instead, the tumor cells disperse and infiltrate into the surrounding brain and are supplied by normal pre-existing capillaries. Should enhancement appear on serial imaging of a known low-grade astrocytoma, it usually indicates transformation to a higher grade tumor.

IDH-mutant astrocytoma is usually in the frontal lobe, sharply demarcated, and often display the "T2-FLAIR mismatch" sign [14]. IDH-wild type astrocytoma often has blurry margins-without the mismatch sign (Fig. 8.7e, f).

IDH-mutant gliomas have a much better prognosis than IDH-wild type. Virtually all (probably all) oligodendrogliomas have IDH mutations - and they enjoy the best prognosis of all diffuse gliomas. Next in survival is the IDH-mutant astrocytoma-about $70 \%$ of lower-grade WHO Gr 2,3 tumors. IDH-wild type astrocytoma (30\% of WHO Gr 2,3) tends to have the same poor prognosis as IDH-wild glioblastomas. In some centers, they are monitored closely for progression and may then be treated like glioblastoma [15].

Oligodendrogliomas present at all ages-with the majority seen in the 4th-6th decades. They are more slowly growing than astrocytoma with a prolonged clinical history that often spans a decade. The long clinical course is often (30$90 \%$ ) associated with dense nodular calcifications (Fig. 8.8). Somewhat different from astrocytoma, they tend to grow toward the cortical surface of the cerebral hemispheres-and they may produce remodeling of the inner table of the skull. Oligodendrogliomas usually have a fine network of capillary vessels, and they may show contrast enhancement, even if they are low grade. Their vascularity may be associated with symptomatic hemorrhage.

Circumscribed gliomas may be intraventricular (ependymoma and choroid plexus neoplasms) or parenchymal. Circumscribed parenchymal gliomas are astrocytic: pilocytic (PA) WHO Gr1; pleomorphic xanthoastrocytoma (PXA); WHO Gr2,3 pilomyxoid astrocytoma (PMA) WHO Gr2; subependymal giant-cell astrocytoma (SEGA) WHO Gr1; or ganglioglioma (GG) WHO Gr1-a mixed glioneuronal neoplasm. Circumscribed astrocytoma usually has mutations in BRAF: BRAF-fusion for infratentorial pilocytic tumors; and BRAF V600E (substitution) for supratentorial PXA, PMA, and GG [16]. SEGA has one of the two mutations associated with tuberous sclerosis: TSC 1 on chromosome 9 or TSC 2 on chromosome 16. Most circumscribed gliomas show avid contrast enhancement. Yet, these neoplasms enjoy a much better prognosis compared to non-enhancing diffuse glio- 


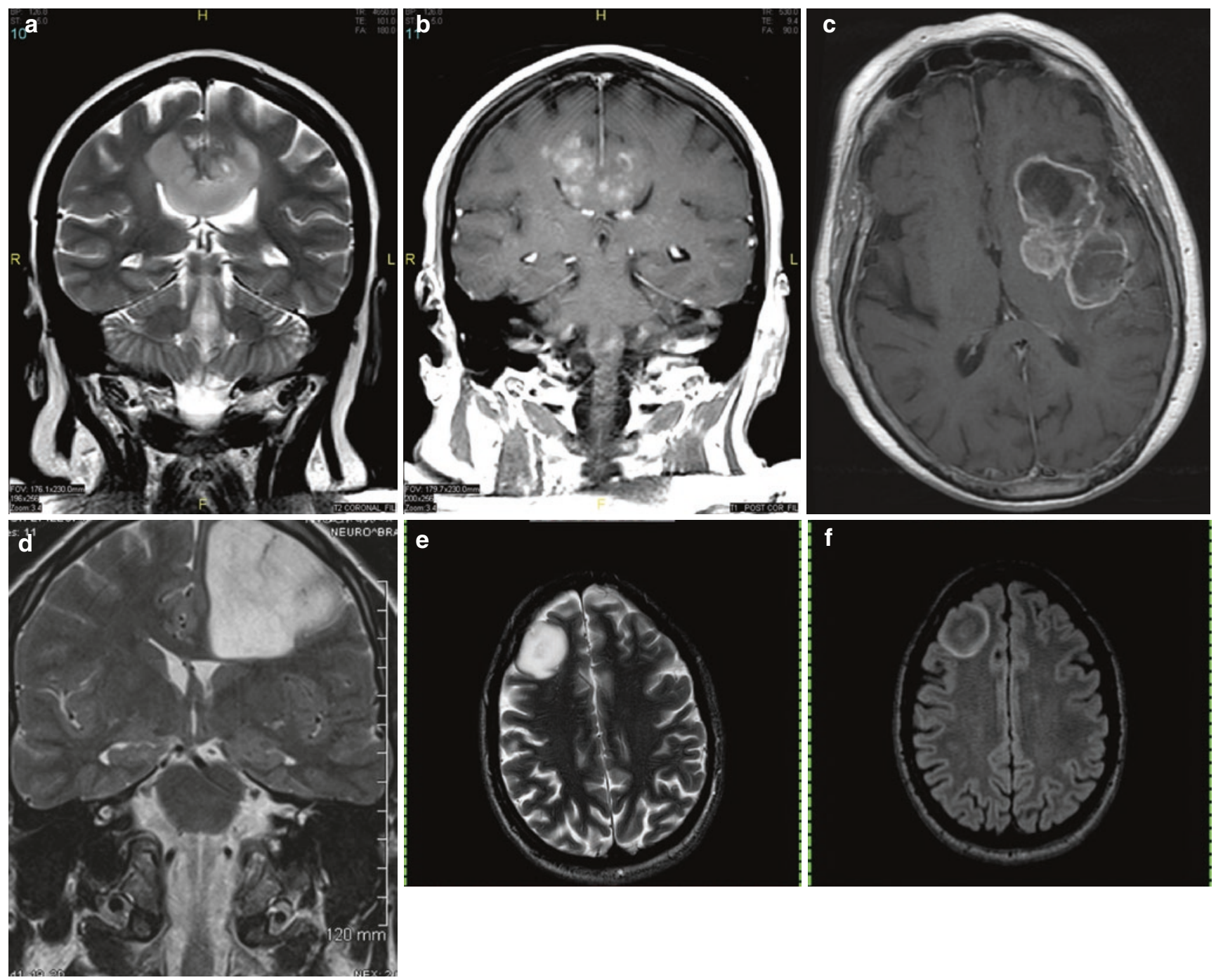

Fig. 8.7 (a, b) Diffuse Glioma a,b Glioblastoma. Coronal T2-weighted MR (a) and T1-weighted after gadolinium (b) demonstrates expansion of the mid-body of the corpus callosum with abnormal hyperintensity. The lesion shows heterogenous patchy enhancement, but without macroscopic necrosis. (c) Glioblastoma WHO Gr4. Axial T1-weighted MR with gadolinium shows a large and heterogenous left insular mass with heterogeneous enhancement. This appearance is "classic" for glioblastoma multiforme with macroscopic areas of necrosis. (d). Astrocytoma WHO Gr2,3. Coronal T2-weighted MR clearly shows abnormal hyper-

mas. The other curiosity is that most BRAF-mutant neoplasms are "fluid-secreting"-producing the characteristic appearance of a solid nodule of enhancement and a homogenous fluid-pool without peripheral enhancement (Fig. 8.9).

Pilocytic astrocytoma is most common in young patients (9-15 years of age) in the cerebellar hemispheres. Pleomorphic xanthoastrocytoma is present in children and mostly younger adults, typically in a superficial location of the cerebral hemispheres. Ganglioglioma usually present with seizures in young adults and most commonly located in the temporal lobe. Pilomyxoid astrocytoma is usually around the third ventricle (Fig. 8.9). intense signal extending toward the surface. This neoplasm has infiltrated and expanded the cerebral cortex, effacing the sulci. This is not vasogenic edema, which usually spares the gray-matter. (e, f) Diffuse astrocytoma WHO Gr 2 (c, d). Axial T2-weighted (c) and FLAIR (d) IDH-mutated diffuse astrocytomas are usually well-demarcated and homogeneous non-enhancing areas of water-like signal-intensity. They often display the "T2-FLAIR mismatch" sign-as shown here, where the center is dark on FLAIR despite hyperintensity on T2

\subsection{Primary CNS Lymphoma}

Whereas the incidence of PCNL in immunocompromised patients has declined following the introduction of effective antiviral therapy, there has been a steady increase of PCNSL in immunocompetent patients.

PCNSL are mostly large B-cell lymphomas and commonly found in the periventricular regions or deep gray nuclei. Owing to their high cellularity, they demonstrate typically restricted diffusion. They enhance strongly with a homogenous enhancement pattern in immunocompetent 

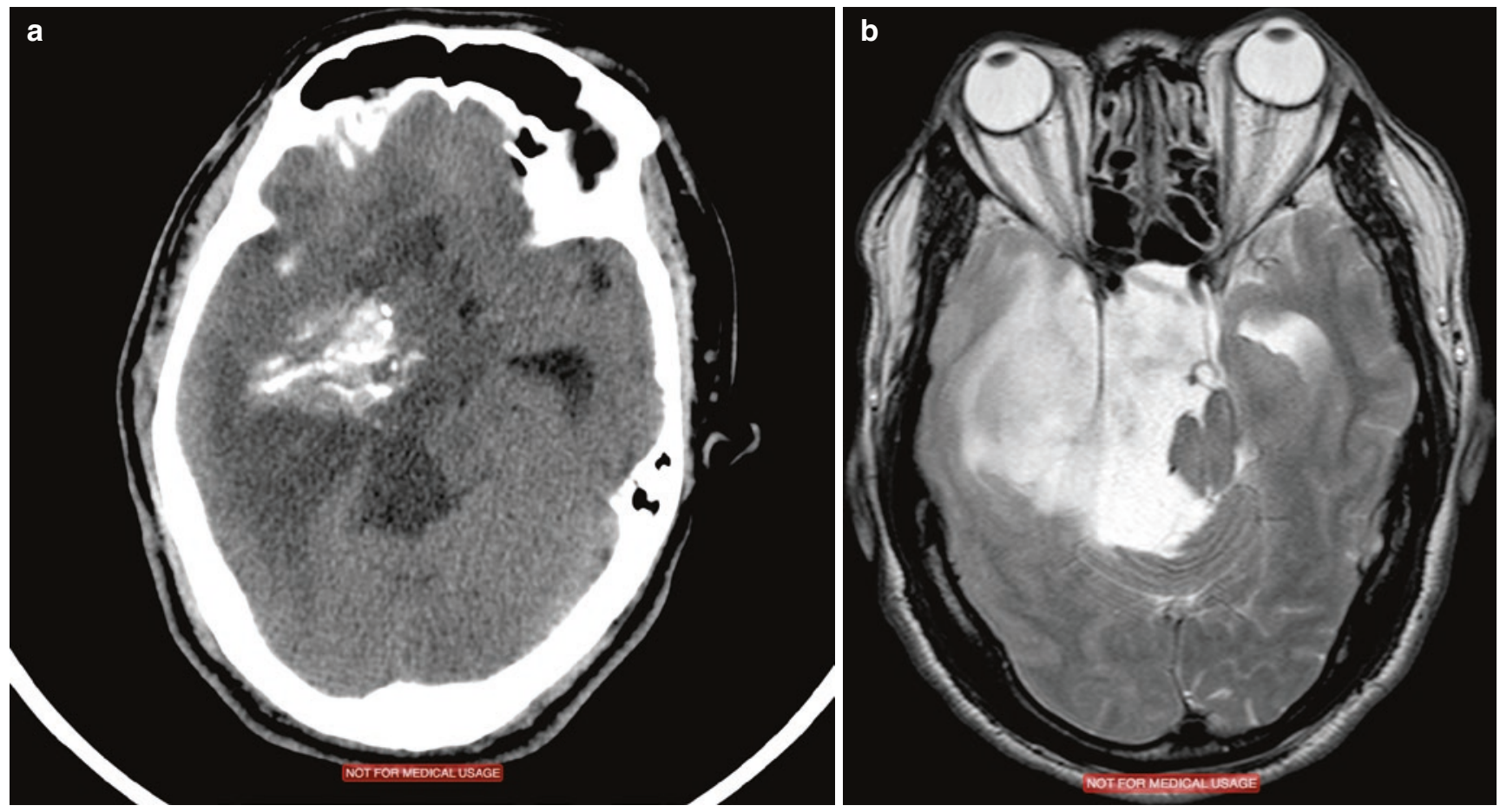

Fig. 8.8 (a, b) Oligodendroglioma WHO Gr2 (same patient as Fig 8.2a). Axial non-contrast CT (a) and T2-weighted MR (b) show a very large area of low attenuation and corresponding high signal-intensity. Surprisingly, the conglomerate hyper-attenuating calcifications do not cause corresponding signal voids on the MR. This tumor was proven to have $1 \mathrm{p} 19 \mathrm{q}$ codeletion by FISH (fluorescent in-situ hybridization) analysis. It was also IDH-mutant, like the majority of diffuse lower-grade gliomas patients and ring enhancement in the immunocompromised. On perfusion imaging, the $\mathrm{rCBV}$ is only minimally increased (much less than, e.g., in glioblastomas), as lymphoma grows angiocentrically and does not stimulate neoangiogenesis.

\section{Key Point}

- Glioblastomas are usually (90\%) IDH-wild type with enhancement and necrosis. Diffuse lower-grade gliomas (WHO Gr2,3) overwhelmingly harbor IDH mutations and do not enhance. The T2-FLAIR mismatch sign indicates an IDH-mutant astrocytoma, distinguishing from oligodendroglioma and IDHwild type astrocytoma. Dense calcifications suggest oligodendroglioma. Circumscribed gliomas usually show prominent contrast enhancement, a fluid component, and have BRAF mutations.

\subsection{Concluding Remarks}

There are multiple tools for triangulating a short differential diagnosis list, and it is important to use "pattern analysis" rather than simple "pattern recognition." Key features include lesion location, relative "volume effect"; secondary vasogenic edema; homogeneity or heterogeneity of the lesion; and patterns of contrast enhancement (homogeneous, patchy, closed or open ring). Multiple lesions usually represent a systemic process: inflammatory; toxic; metabolic; genetic; or hematogenous dissemination. Extraaxial masses are nonglial, while intraaxial neoplasms may be metastatic from extra-CNS sources, or primary gliomas.

\section{Take Home Messages}

- Lesions with vasogenic edema should also demonstrate contrast enhancement

- Solitary deep lesions are more likely to be primary gliomas

- Round nodular lesions at the gray-white junction are more likely to be metastatic

- Diffuse gliomas affect the cortex, vasogenic edema does not

- IDH-mutant diffuse astrocytoma shows T2-FLAIR mismatch sign

- Extraaxial lesions are meningioma, schwannoma, and congenital cysts 

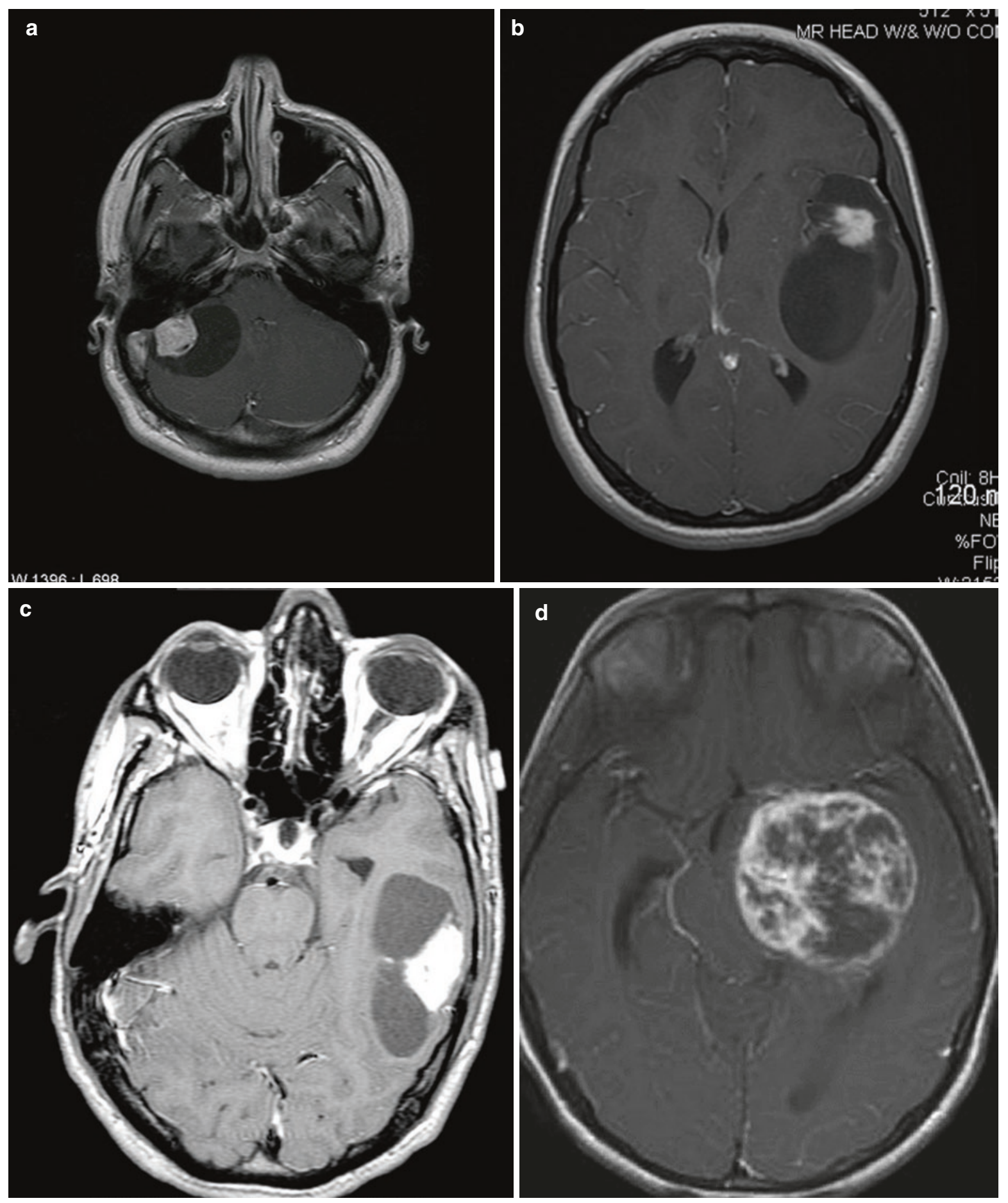

Fig. 8.9 (a, b, c, d) BRAF-mutated circumscribed astrocytoma. Axial T1-weighted MR after gadolinium: (a) pilocytic astrocytoma; (b) pleomorphic xanthoastrocytoma; (c) ganglioglioma; and (d) pilomyxoid astrocytoma. Fluidsecreting neoplasms usually show contrast enhancement limited to a nodule with a peripheral puddle of fluid $(\mathbf{a}-\mathbf{c})$. The absence of a complete rim of enhancement is strongly suggestive of a "fluid-secreting" circumscribed glioma - rather than necrosis in a highgrade tumor. However, in some cases (d), fluid producing tumors can show extremely heterogeneous patterns of enhancement- and may mimic an aggressive and necrotic tumor. The fluid attenuation and signal-intensity may deviate from CSF usually due to protein and rarely from hemorrhage 


\section{References}

1. Smirniotopoulos JG, Murphy FM, Rushing EJ, Schroeder JW. Patterns of contrast enhancement in the brain and meninges. Radiographics. 2007;27(2):525-51.

2. Kim DS, Na DG, Kim KH, Kim JH, Kim E, Yun BL, et al. Distinguishing tumefactive demyelinating lesions from glioma or central nervous system lymphoma: added value of unenhanced CT compared with conventional contrast-enhanced MR imaging. Radiology. 2009;251(2):467-75.

3. Suh CH, Kim HS, Jung SC, Choi CG, Kim SJ, Kim SJ. MRI findings in tumefactive demyelinating lesions: a systematic review and meta-analysis. AJNR Am J Neuroradiol. 2018;39(9):1643-9.

4. Suthiphosuwan S, Sati P, Guenette M, Montalban X, Reich DM, Bharatha A, et al. The central vein sign in radiologically isolated syndrome. AJNR Am J Neuroradiol. 2019;40(5):776-83.

5. Nussbaum ES, Djalilian HR, Cho KH, Hall WA. Brain metastases. Histology, multiplicity, surgery, and survival. Cancer. 1996;78(8):1781-8.

6. Hwang TL, Close TP, Grego JM, Brannon WL, Gonzales F. Predilection of brain metastasis in gray and white matter junction and vascular border zones. Cancer. 1996;77(8):1551-5.

7. Lyndon D, Lansley JA, Evanson J, Krishnan AS. Dural masses: meningiomas and their mimics. Insights Imaging. 2019;10(1):11.

8. Lee KJ, Joo WI, Rha HK, Park HK, Chough JK, Hong YK, et al. Peritumoral brain edema in meningiomas: correlations between magnetic resonance imaging, angiography, and pathology. Surg Neurol. 2008;69(4):350-5
9. Osawa T, Tosaka M, Nagaishi M, Yoshimoto Y. Factors affecting peritumoral brain edema in meningioma: special histological subtypes with prominently extensive edema. J Neuro-Oncol. 2013;111(1):49-57.

10. Crisi G. H MR spectroscopy of meningiomas at 3.0T: the role of glutamate-glutamine complex and glutathione. Neuroradiol J. 2011;24(6):846-53.

11. D'Haese S, Parmentier H, Keppler H, Van Vooren S, Van Driessche $\mathrm{V}$, Bauters W, et al. Vestibular schwannoma: natural growth and possible predictive factors. Acta Otolaryngol. 2019;139(9):753-8.

12. Andronesi OC, Rapilino O, Gerstner E, Chi A, Batchelor T. Detection of oncogenic IDH1 mutations using magnetic resonance spectroscopy of 2-hydroxyglutarate. J Clin Invest. 2013;123(9):3659-63.

13. Wen PY, Reardon DA. Neuro-oncology in 2015: progress in glioma diagnosis, classification and treatment. Nat Rev Neurol. 2016;12(2):69-70.

14. Suh CH, Kim HS, Jung SC, Choi CG, Kim SJ. Imaging prediction of isocitrate dehydrogenase (IDH) mutation in patients with glioma: a systemic review and meta-analysis. Eur Radiol. 2019;29(2):745-58.

15. Eckel-Passow JE, Lachance DH, Molinaro AM, Walsh KM, et al. Glioma groups based on $1 \mathrm{p} / 19 \mathrm{q}$, IDH, and TERT promoter mutations in tumors. N Engl J Med. 2015;372(26):2499-508.

16. Maraka S, Janku F. BRAF alterations in primary brain tumors. Discov Med. 2018;26(141):51-60.

Open Access This chapter is licensed under the terms of the Creative Commons Attribution 4.0 International License (http://creativecommons. org/licenses/by/4.0/), which permits use, sharing, adaptation, distribution and reproduction in any medium or format, as long as you give appropriate credit to the original author(s) and the source, provide a link to the Creative Commons license and indicate if changes were made.

The images or other third party material in this chapter are included in the chapter's Creative Commons license, unless indicated otherwise in a credit line to the material. If material is not included in the chapter's Creative Commons license and your intended use is not permitted by statutory regulation or exceeds the permitted use, you will need to obtain permission directly from the copyright holder. 